\author{
A.N. Malaviya ${ }^{\mathrm{a}, \mathrm{b}}$ \\ Suad Abdeend ${ }^{\mathrm{c}}$ \\ M.A.F. Qurtom ${ }^{\mathrm{b}}$ \\ S. Al-Ghuriear ${ }^{\mathrm{b}}$ \\ H.I. Kablawi ${ }^{\mathrm{b}}$ \\ a Department of Medicine, Faculty \\ of Medicine, Kuwait University, \\ and Departments of \\ ${ }^{b}$ Medicine, and \\ c Pathology, Mubarak Al-Kabeer \\ Hospital, Ministry of Health, \\ Kuwait
}

\section{Erythema nodosum or Erythema induratum: An Important Distinction for Appropriate Treatment}

\begin{abstract}
Objective and Importance: The objective of this case report is to draw the attention of the readers to a commonly seen disorder, namely panniculitis. Clinically, the condition resembles cellulitis, that often causes a diagnostic problem. The report hightlights the importance of accurate diagnosis of panniculitis and its subtypes for its proper management. Clinical Presentation: Two patients with clinically typical lesions of panniculitis on their lower extremities are described. The lesions in these 2 patients had marked clinical resemblance to cellulitis. However, with the help of biopsy and histopathological examination it was possible to accurately diagnose the distinct forms of panniculitis in these patients. It helped in the correct management of the patients with satisfactory outcome. Conclusion: It is concluded that in patients with lesions resembling cellulitis, especially on the shin, the possibility of panniculitis must be considered. The experience with these 2 patients highlights the need for accurate histological characterization as an essential step in the diagnosis and proper management of different forms of panniculitides.
\end{abstract}

\section{Key Words}

Panniculitis

Erythema induratum

Bazin's disease

Erythema nodosum nodosum (EN), a form of septal panniculitis without vasculitis, is the commonest variety seen in clinical practice [1-6]. It is a frequently diagnosed condition in Kuwait [7, 8]. However, the clinical and morphologic picture of the lesion may not be specific of this condi-

\begin{tabular}{ll}
\hline KARGER & @ 1998 S. Karger AG, Basel \\
Fax +41 61 306 12 34 & 1011-7571/98/0074-0298\$15.00/0 \\
$\begin{array}{l}\text { E-Mail karger@karger.ch } \\
\text { www.karger.com }\end{array}$ & Accessible online at: \\
& http://BioMedNet.com/karger
\end{tabular}

Prof. A.N. Malaviya, MD, FACP, FACR

Department of Medicine, Faculty of Medicine Kuwait University, PO Box 24923

13110 Safat (Kuwait)

Fax +965 533 8907, E-Mail<anand@hsc.kuniv.edu.kw> 
tion and some of the patients may have other forms of panniculitis including erythema induratum (EI), a form of panniculitis associated with vasculitis of medium- or small-sized arteries or veins in the panniculus, in conjunction with an adjacent suppurative and/or granulomatous panniculitis [4], a histology believed to be rather specific for tuberculosis [1-6]. This disease has often been called 'Bazin's disease' after the name of the person who described it for the first time [cited in ref. 8].

In this report, we present 2 patients who had lesions on the legs associated with constitutional and rheumatic symptoms that were first considered EN. One of them suffered protracted fluctuating illness due to lack of accurate early diagnosis. However, the histological study of the lesion in both of them showed features of EI that led to the diagnosis of tuberculosis. With appropriate treatment, both the patients recovered from their illness. This report highlights the fact that for achieving proper diagnosis and treatment in patients with any form of panniculitis, histopathological categorization of the lesion may be necessary.

\section{Case Reports}

\section{Case 1}

A 33-year-old Indian man presented with a history of an unusual skin lesion on his legs of 2-year duration. In the beginning, he noticed vague pains in the lower part of his legs associated with mild constitutional symptoms including muscular aches and pains, mild arthralgias, with occasional feverish feeling. He was treated symptomatically and felt better. However, soon he noticed a few reddish-purplish nodules on the legs that were tender. Lesions quickly increased in number, became bigger and a few of them ulcerated. Over the next several months he consulted a number of clinics and hospitals. A diagnosis of chronic nodularulcerative skin condition was made, and he was given symptomatic and antibiotic treatment without definite relief. The constitutional symptoms became worse. He continued with fluctuating constitutional symptoms

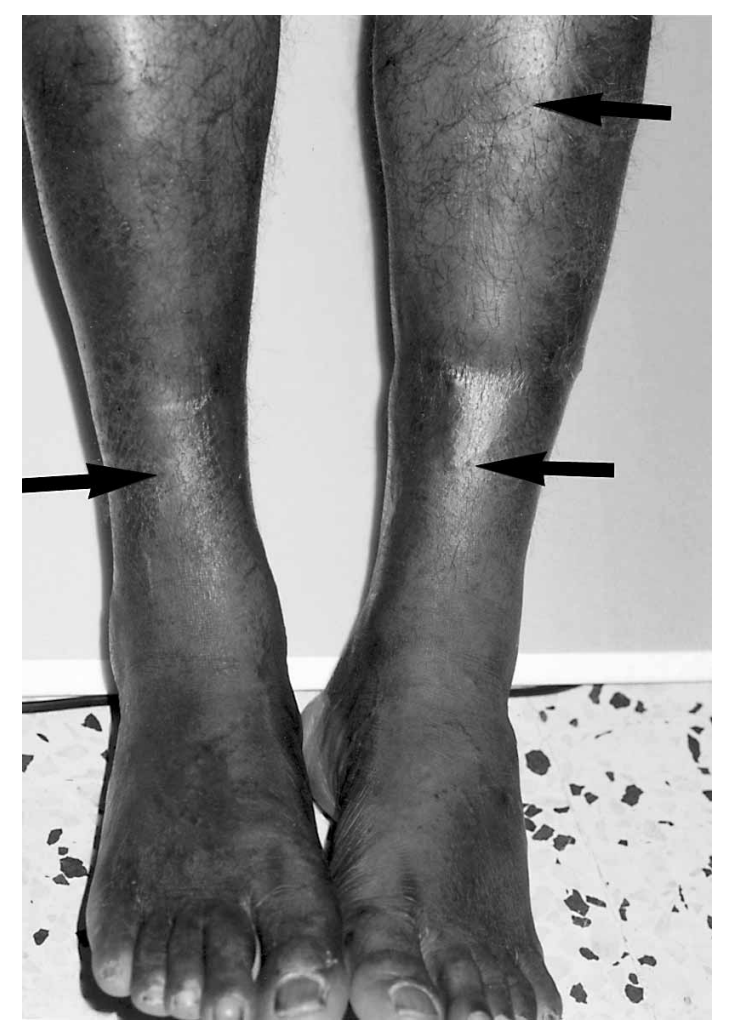

Fig. 1. Photograph of the legs showing skin nodules with ill-defined margins merging with each other with hyperpigmented, stretched and shiny overlying skin with a distinct erythematous-purplish hue. Some scarring of the healed ulcerated nodules is also visible.

and leg ulcers that healed on a few occasions, but reappeared soon. This process went on for almost 2 years. However, because of exacerbation in the nodulo-ulcerative skin condition with increasing constitutional symptoms and vague 'rheumatic pains' he was referred to this hospital for opinion. On examination there were a few palpable discrete nontender 0.5 - to $1-\mathrm{cm}$-size lymph nodes in the left axilla. There were bilateral skin nodules, about 3-4 on each leg. Margins of the nodules were ill-defined and appeared to merge with each other. The skin over the nodules was hyperpigmented with a distinct erythematous-purplish hue and appeared stretched and shiny. There were scars over some of the nodules while a few of them had superficial chronic nonhealing ulcers (fig. 1). Routine investigations showed the following results: ESR $40 \mathrm{~mm}$ 
Fig. 2. Epithelial cells (arrows) at the periphery of the granulomas invading the subcutaneous fat. Lymphocytes are seen at the outside of the lesion. HE. $\times 200$.

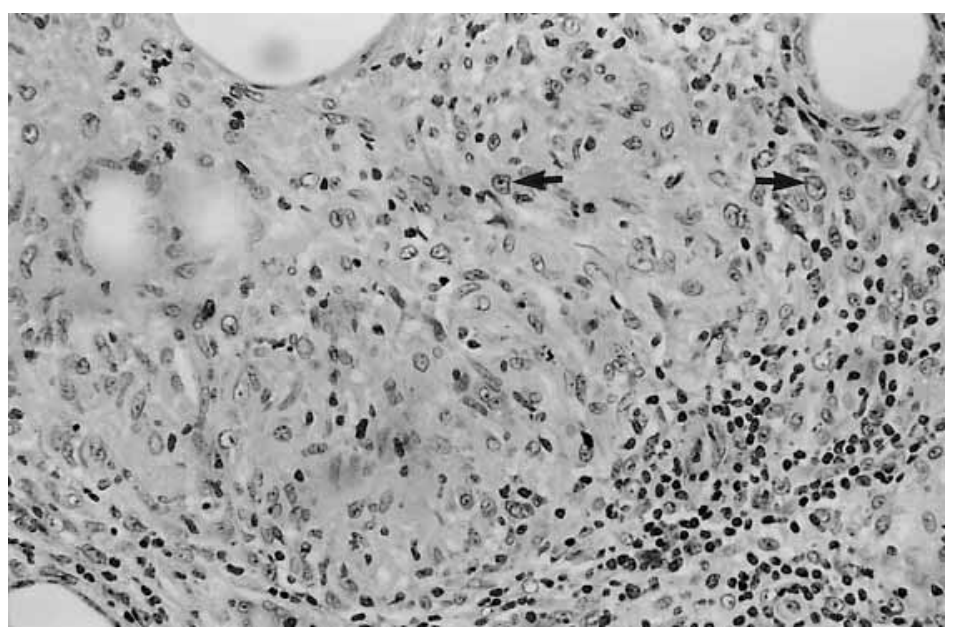

Fig. 3. Fibrinoid necrosis of the wall of a vein with inflammatory cells and necrotic debris within an area of panniculitis. HE. $\times 100$.

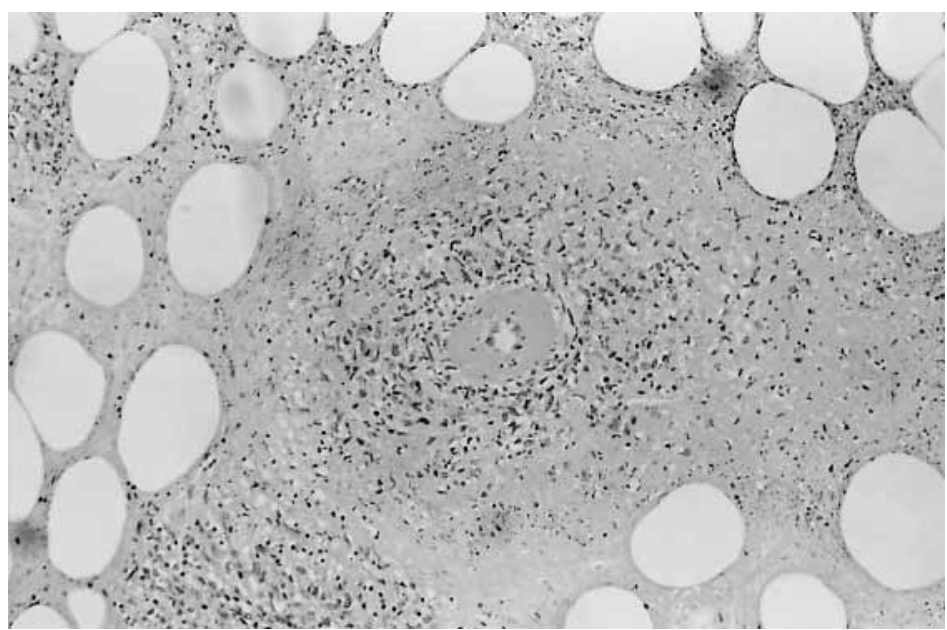

1 st hour (normal range in males $>50$ years $0-20$ ), normal complete blood count, normal serum biochemistry including liver enzymes, renal profile and serum proteins, normal immunological tests including rheumatoid factor, antinuclear antibody, C-reactive protein, and cryoproteins. Screening for hepatitis B and C was negative. Biopsy of the subcutaneous nodules along with the overlying skin was carried out. Histopathology showed the main lesion in the lower dermis and subcutis, consisting of lobular panniculitis with confluent areas of necrotizing granulomas comprising of epitheloid cells (fig. 2), and occasional Langerhans giant cells. The granulomas were surrounding necrotic adipocytes. There was necrotizing vasculitis affecting some vessels characterized by fibrinoid necrosis of the vessel wall (fig. 3), showing infiltration with mononuclear nuclear inflammatory cells. The surrounding tissue showed suppurative panniculitis in which neutrophils and debris were present within the necrotic fat (fig. 4). Acid-fast stains were, however, negative for acid-fast bacilli.

The patient was given standard triple-drug antituberculous therapy consisting of isoniazid, rifampin and pyrazinamide. He tolerated medicines well and his liver function tests remained normal during the followup. In about 3 weeks he noticed improvement in the constitutional symptoms, and the lesions on the legs started to heal in about 5-6 weeks. By 3 months the leg 
Fig. 4. Neutrophils are seen in the area of panniculitis giving rise to suppurative changes. HE. $\times 200$.

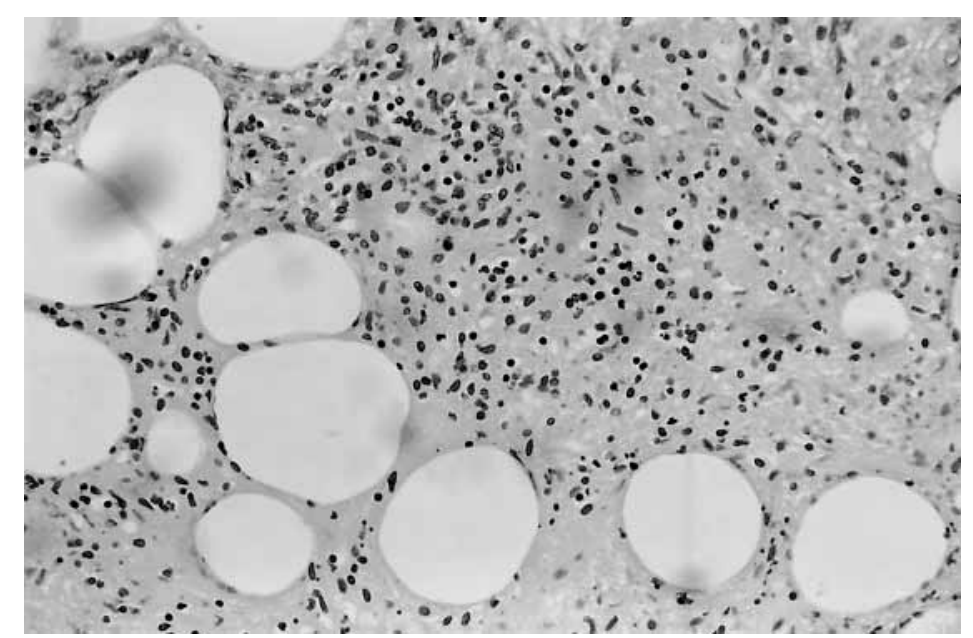

Fig. 5. Photograph of the legs showing sparse erythematous elevated skin nodules over both shins (arrows) that were tender.

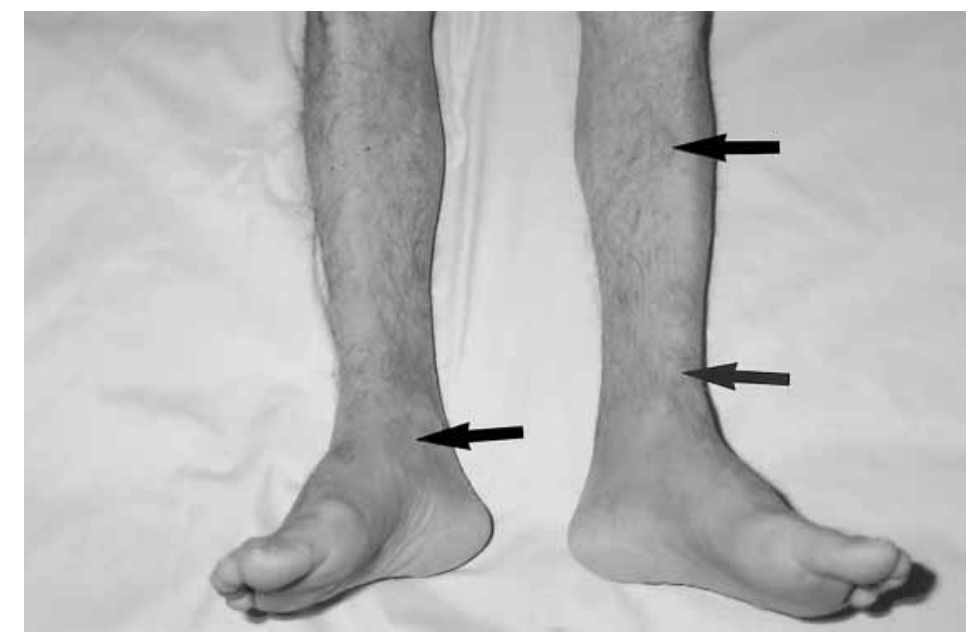

lesions had completely healed. However, the pigmentation and the stretched shiny appearance of the overlying skin persisted. The medicines were discontinued after a total of 9 months of treatment. Till the time of this writing the patient had completed a follow-up of 9 months since the medicines were discontinued, and remained in completely normal health.

\section{Case 2}

A 50-year-old Iranian man presented with a 4-week history of constitutional symptoms consisting of fever, night sweats, marked fatigue, loss of appetite and $10 \mathrm{~kg}$ weight loss. Over the last 3 days he had noticed painful

Erythema nodosum versus

Erythema induratum raised nodular reddish lesions on his shins. Simultaneously with the appearance of the lesions on the legs, he also noted painful swellings in his ankles. He had a past history of brucellosis diagnosed and treated in Iran 24 years ago. Positive physical findings included fever $\left(38.4^{\circ} \mathrm{C}\right)$, emaciation, nontender matted lymph nodes $1-2 \mathrm{~cm}$ in size in both cervical areas, follicular tonsillitis, and sparse tender erythematous elevated skin nodules over both the shins (fig. 5). Both the ankles were mildly swollen and tender. Abdominal examination showed a 16-cm tender smooth hepatomegaly. The spleen was not palpable. Investigations showed the following results: WBC 19 (normal range $4-10) \times 10^{9} / 1$ with differential count granulocytes

Med Principles Pract 1998;7:298-305 
Fig. 6. Vasculitis with marked inflammation with neutrophils many of which have broken down to nuclear debris. HE. $\times 200$.

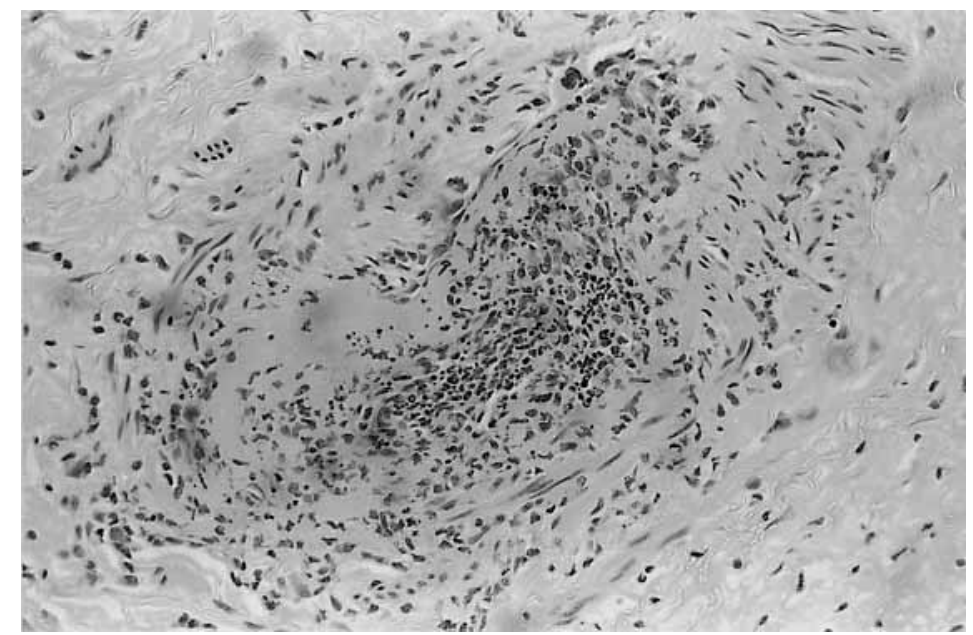

Fig. 7. Multiple epitheloid cell granulomas (arrows) surrounding fat cells within the areas of panniculitis. HE. $\times 200$

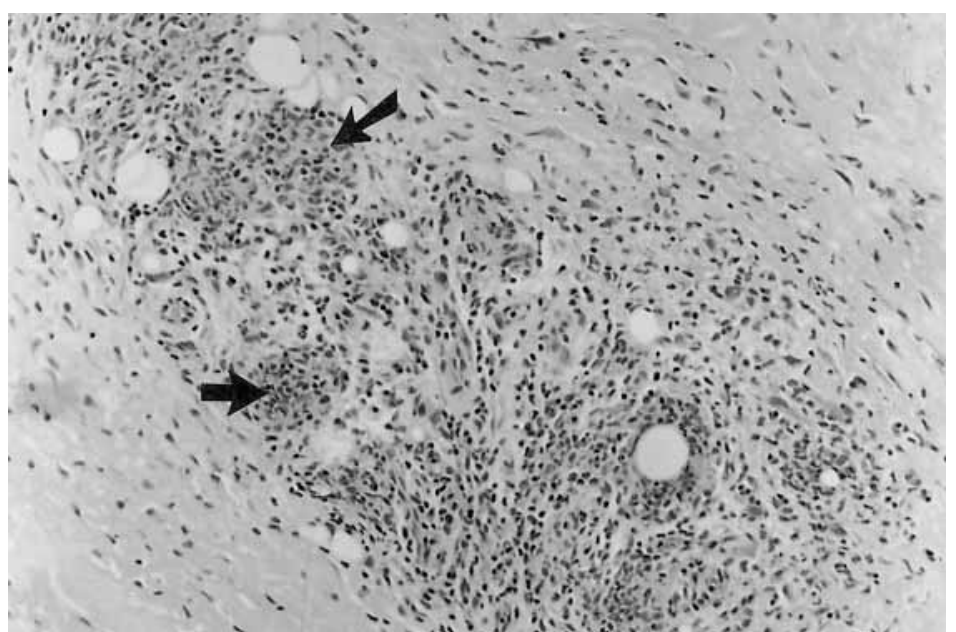

$80 \%$ (normal range 45-74), lymphocytes $15 \%$ (normal range 16-45), monocytes $0.4 \%$ (normal range 4-10), hemoglobin $110 \mathrm{~g} / 1$ (normal range 130-118), platelets $428 \times 10^{9} / 1$ (normal range 130-400), ESR $120 \mathrm{~mm}$ 1 st hour (normal range in males $>50$ years $0-20$ ). The routine and microscopic urine examination was normal. The serum biochemistry including liver enzymes, renal profile and serum proteins were normal except alkaline phosphatase that was 146 units/l (normal range 2-120), serum albumin $25 \mathrm{~g} / \mathrm{l}$ (normal range 3555), total serum globulin $35 \mathrm{~g} / \mathrm{l}$ (normal range 20-30). Coagulation profile was normal. Immunological tests including rheumatoid factor, antinuclear antibody and C-reactive protein tests were negative. Blood, urine, sputum and throat swab smears and cultures did not show positive tests for any pathogens. Blood smears were negative for any parasites. Sputum culture for acid-fast bacilli was also negative. Virological studies including those for cytomegalovirus (specific IgM and IgG antibodies) and Epstein-Barr virus (specific IgM and $\mathrm{IgG}$ antibodies and 'Monospot test') yielded negative results. Brucella agglutination test was negative. Echocardiogram was normal. Abdominal ultrasound examination and plain roentgenogram of the chest was normal. A Mantoux test with 1 unit purified-protein derivative gave a strongly positive reaction of $37 \times$ $37 \mathrm{~mm}$ of induration after $48 \mathrm{~h}$. Screening for hepatitis $\mathrm{B}$ and $\mathrm{C}$ was negative. Biopsy of the subcutaneous nod- 
ules along with the overlying skin was carried out. The histopathology showed focal leukocytoclastic vasculitis (fig. 6) involving small arteries and veins characterized by fibrinoid necrosis of the vessel wall with mononuclear inflammatory cell infiltration and nuclear debris. There was thrombotic occlusion within the lumen of these vessels. There were small foci of panniculitis with epitheloid cell granulomas (fig. 7). Foreign-body type of giant cells were seen around sutural material. The surrounding dermal collagen showed areas of fibrinoid necrosis. Mild mononuclear inflammatory infiltrate was present around some of the skin appendages. The histological features were those of leukocytoelastic vasculitis and papulonecrotic tuberculid. Lymph node biopsy showed typical features of tuberculous lymphadenitis with multiple large confluent epitheloid cell granulomas and Langerhans giant cells, and it tested positive for acid-fast bacilli on special stains.

The patient was given triple-drug antituberculous therapy consisting of isoniazid, rifampin and pyrazinamide. He tolerated the medicines well. Over a period of 2 weeks he started to gain weight and the fever subsided. At the time of discharge the investigations showed ESR $90 \mathrm{~mm} \mathrm{Hg}$, hemoglobin $122 \mathrm{~g} / \mathrm{l}$, WBC $10.4 \times 10^{9} / 1$, granulocytes $69 \%$, lymphocytes $22 \%$, platelets $428 \times 10^{9} / 1$. Liver function monitoring did not show any abnormality. At the follow-up visit 4 weeks after discharge, he was found to have improved in his general health and felt normal. He had not repeated the investigations that were suggested and informed that he was leaving for his home country. He was advised to continue medicines for a minimum of 6-9 months and to be under the care of a local doctor for proper follow-up.

\section{Discussion}

The 2 cases presented in this report illustrate the problems in the diagnosis of panniculitis. What clinically appears as panniculitis may necessitate histological categorization. Although EN, a 'septal panniculitis without vasculitis', is the most common form, the morphological picture may not be specific for this condition. The lesion could be due to any of the several underlying conditions, including EI, a 'lobular panniculitis with vasculitis', that is often associated with an underlying focus of tuberculosis [1-6]. The examples of these patients highlight the need for histopathological study that, in these 2 cases, led to the diagnosis of mycobacterial infection. Therefore, histopathology is recommended for achieving the proper diagnosis and treatment in those patients where the cause of panniculitis is not obvious.

Panniculitis is a generic term used for inflammation in the subcutaneous fatty tissue of the body [9]. Most authorities now classify panniculitides into four categories based upon histopathological criteria, namely: (i) septal panniculitis without vasculitis; (ii) lobular panniculitis without vasculitis; (iii) mixed septal and lobular panniculitis - but without vasculitis, and (iv) panniculitis with vasculitis $[1,2]$. Clinically the lesions of different varieties of panniculitides may look alike with nodular indurated erythematous inflamed areas in the subcutaneous tissue. The overlying skin may show different features in different stages of the lesions. Thus, the skin may appear inflamed, or it may have a brawny indurated appearance, or it may appear ecchymotic, or become scaly when healing. However, there are certain features that help in clinical distinction between different categories of panniculitides. These include: (i) sex of patient - EN is predominantly a disease of women, if seen in men, Lofgren's syndrome (acute sarcoidosis with bilateral hilar lymph nodes, EN-type lesions on shin and inflammatory peripheral arthritis) or Behçet's disease are the most likely underlying cause [1]; Vilanova's disease, a chronic relapsing form of EN (also called erythema migrans chronicum [1]), is seen only in women. The other features include: (ii) appearance and feel of the lesion; (iii) number and distribution of the lesions (EN lesions are almost entirely limited to shin); (iv) presence or absence of ulceration (EN lesions never ulcerate while those in other forms of panniculitis may ulcerate), and (v) scarring (a prominent feature of panniculitis 
associated with Behçet's disease). Increased awareness of the distinguishing clinical features between different forms of panniculitides would help in deciding in whom histopathological categorization is necessary for achieving proper diagnosis.

Among the various categories of panniculitides, EN is the commonest form seen in clinical practice [1-6]. Therefore, characteristic clinical features of EN may be taken as a reference point. Deviation from this clinical pattern should be a guide for obtaining histopathological confirmation of the actual category of panniculitis. Characteristic clinical features of EN are a predominance of this in young females and an acute appearance of one or a few tender erythematous nodules on the anterior tibial surface that are better palpated than visualized. Overlying skin appears inflamed and feels indurated. As the lesions age, the nodules soften and become less palpable, a peculiar ecchymotic purplish discoloration appears and scaling of the skin may become prominent. (It is not uncommon for such patients to get the label of 'cellulitis' and to receive antibiotics empirically.) Over the next 4-6 weeks the lesions eventually heal without suppuration or scarring. Although EN is usually an acute process, as discussed above, a chronic relapsing variant of EN is not uncommon. It is much more common in middleaged women [1,2]. Clinically, the lesions look very similar to those seen in the acute condition. However, ankle arthritis (unilateral or bilateral) is a unique feature associated in many of these cases [5]. This type of arthritis may sometimes appear a few days before the appearance of the EN nodules.

In contrast, the clinical features of EI include occurrence without any sex predilection, superficial resemblance of the lesions to EN occurring predominantly on the anterior tibial region, but the lesions may occur at other sites in the body, often with ulceration and suppuration, and healing with scarring. However, the actual diagnosis is made on a constellation of histological features consisting of vasculitis of medium- or small-sized arteries or veins in the panniculus in conjunction with an adjacent suppurative and/or granulomatous panniculitis [4]. In both the cases reported here, the clinical features were against the diagnosis of EN. Thus in case 1, the lesions had shown repeated ulcerations and extensive scarring in the lower regions of both legs, features not seen in EN (fig. 1). In case 2, the nodules had extended up to the midthighs, a most unusual occurrence for lesions of EN. Of course, the diagnosis of EI was established on histological examination showing the characteristic histopathology in both cases (fig. 2-4, 6, 7). In case 1, a skin and subcutaneous tissue biopsy provided the essential clue to tuberculosis as the etiology of the underlying disorder. Had the biopsy not been performed, tuberculosis might not have been recognized even at this late stage. Moreover, there is a tendency among clinicians to give corticosteroids in recalcitrant skin lesions especially if they appear to be due to some form of immunological reaction. EN is one such lesion where steroids are often used if the lesion becomes chronic or recurrent. Had empiric corticosteroid therapy been prescribed to these patients, an exacerbation of their mycobacterial infection could have resulted.

In many cases with EI, it may not always be easy to demonstrate the causative agent, i.e. Mycobacterium tuberculosis. Therefore, it was fortunate that in both the cases presented here, it was possible to diagnose tuberculosis with a fair degree of certainty. In case 1 , the typical histopathology along with the therapeutic response that was confirmed in the prolonged follow-up left no doubt on the diagnosis of tuberculosis. In case 2, acid-fast bacilli were demonstrated in the lymph node biopsy. This is important because Whitfield has de- 
scribed almost identical patients without any evidence of tuberculosis [cited in ref. 8]. For this reason, the modern view is to call such lesions 'nodular vasculitis' representing a hypersensitivity reaction manifested by medium-sized vessel vasculitis in which the adjacent panniculus becomes involved in the inflammatory process and becomes necrotic. Once the diagnosis of nodular vasculitis is made histologically, all efforts should be made to look for the evidence of tuberculosis. In this regard, the availability of a polymerase chain reaction has been reported to be of help in improving the diagnosis of tuberculosis in those cases where smears and cultures may be negative [10]. Unfortunately, this could not be done in case 1 due to some practical difficulties.

In summary, panniculitis, i.e. inflammatory lesions of the panniculus, is common in clinical practice and it entails a wide spectrum of differential diagnosis. EN being the commonest form of panniculitis seen in practice, there is a tendency among clinicians to label all forms of panniculitides as EN. The present report emphasizes the importance of using the generic term 'panniculitis' for most such patients until careful clinical examination, often combined with histopathological and microbiological studies, had established the exact diagnostic category of panniculitis. This approach is recommended in order to avoid using certain drugs (e.g. steroids) empirically that can have deleterious effects on the clinical outcome.

\section{Acknowledgment}

The help provided by Ms. Lynn Marie Lund, Lecturer, Department of English, Faculty of Allied Health Sciences, Kuwait University, in the preparation of the manuscript, is gratefully acknowledged.

\section{References}

1 Callen JP, Zax RH: Panniculitis; in Maddison PJ, Isenberg DA, Woo P, Glass DN (eds): Oxford Textbook of Rheumatology, ed 2. Oxford, Oxford University Press, 1993, pp 918-922.

2 Callen JP: Panniculitis; in Klippel JH, Dieppe PA (eds): Rheumatology. London, Mosby-Year Book Europe, 1994, 6.24.1-4.

3 George DL: Arthritis with skin and nail changes; in Klippel JH, Dieppe PA (eds): Rheumatology. London, Mosby-Year Book Europe, 1994, pp 2.2.1-2.2.14.

4 Patterson JW: Differential diagnosis of panniculitis. Adv Dermatol 1991; 6:302-329.
5 Soter NA, Franks AG Jr: The skin and rheumatic diseases; in Kelly W, Harris E Jr, Ruddy S, Sledge C (eds): Textbook of Rheumatology, ed 4. Philadelphia, Saunders, 1993, pp 519-530.

6 Moschella S, Hawley H: Dermatology, ed 3. Philadelphia, Saunders, 1992, pp 1098-1099.

7 Malaviya AN, Al-Awadi A, Al-Saeid K, Al-Jarallah K, Shehab D: Letter from Kuwait. Br J Rheumatol 1996; 35:380-381.
8 Rayan TJ: Cutaneous vasculitis; in Chapino RH, Burton JL, Ebling FJG (eds): Textbook of Dermatology, ed 5. Cambridge, Blackwell Scientific Publications, 1992, pp 1931-1944.

9 Patterson JW: Panniculitis: A guide to differential diagnosis. J Assoc Military Dermatol 1983;9:36-39.

10 Penneys NS, Leonardi CL, Cook S, Blauvelt A, Rosenberg S, Eells LD, Konwiser M, Aaronson CM: Identification of Mycobacterium tuberculosis in DNA in five different types of cutaneous lesions by the polymerase-chain reaction. Arch Dermatol 1993;129:1594-1598. 